\title{
Investigación en Progreso: Desarrollo de Modelos y Algoritmos del Campo de la Robótica Basado en un Enfoque de Tecnologías Inteligentes
}

\author{
Alejandro Hossian, Lilian Cejas, Roberto Carabajal, César Echeverría, Maxiliano Alvial, Verónica Olivera \\ Proyecto de Investigación UTI3536 de la Facultad Regional Neuquén. Universidad Tecnológica Nacional. Argentina. \\ alejandrohossian@yahoo.com.ar
}

\begin{abstract}
Resumen - La propuesta del presente proyecto es el desarrollo de distintas arquitecturas de software ejecutándose en diversas plataformas de hardware que permitan definir los comportamientos de robots autónomos y manipuladores en base a un enfoque cognitivo. Se pretende que el robot posea un comportamiento deseado en función a las prestaciones requeridas al mismo. Este comportamiento deseado podrá evaluarse a través de distintos indicadores que reflejarán la aptitud del agente robótico para llevar a cabo las tareas y conductas que le son requeridas, en particular cuando interactúa en entornos dinámicos y cambiantes. Con el objeto de lograr este propósito de relacionar adecuadamente arquitecturas software $y$ plataformas de hardware con distintos ambientes y comportamientos deseados en función de las prestaciones requeridas, se explorará el uso de diferentes recursos tecnológicos para el diseño de la estructura cognitiva del robot. Entre los soluciones tecnológicas a emplear en el diseño del cerebro del robot se encuentran Redes Neuronales Artificiales, Lógica Difusa, Sistemas Expertos, Aprendizaje Automático, algoritmos genéticos. Asimismo, el proyecto explorará el diseño de arquitecturas cognitivas híbridas que combinen e integren adecuadamante las tecnologías previamente enunciadas, procurando brindar recomendaciones para la construcción de un módulo inteligente de robot que optimice su comportamiento global en términos de las prestaciones requeridas, para determinadas plataformas de hardware y para distintas configuraciones de entornos de navegación robótica tanto estáticos como dinámicos.
\end{abstract}

Palabras claves-Robótica, Software, Hardware, Tecnologías Inteligentes, Entornos de navegación.

\section{JustificAción De LA Propuesta}

La globalización de los mercados productivos ha generado un ambiente altamente competitivo, dando como resultado la modificación y en algunos casos el desarrollo de nuevas tecnologías. Para mantener esquemas de producción rentables, las grandes compañías se han visto en la necesidad de optimizar sus procesos de manufactura. Esto ha creado la demanda de recursos humanos altamente capacitados $\mathrm{y}$ enfocados a la mejora continua de los procesos industriales en general.

En este contexto se plantea la necesidad de abordar nuevas perspectivas desde las Tecnologías Inteligentes a los efectos de responder a este nuevo paradigma de los mercados competitivos.

\section{Estado ACtuAl Del Conocimiento Sobre El Tema}

El entorno real para los robots autónomos es dinámico e impredecible. Esto causa que, para la mayor parte de los dominios, tener una teoría del dominio (modelo) perfecta de cómo afectan las acciones del robot el entorno es un ideal.
Se conocen dos perspectivas mayoritarias entre los diferentes tipos de arquitecturas para el control de robots autónomos a lo largo de los últimos años. Por un lado se encuentran las arquitecturas reactivas, como la observada en la figura 1, que proponen la consecución de metas complejas por parte del robot basándose únicamente en un conjunto de reacciones simples. Estas reacciones simples reciben distintos nombres: módulos, agentes, agencias, controladores o comportamientos.

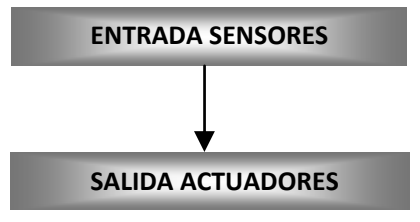

Fig. 1. Arquitectura reactiva.

Por otro lado se encuentran las arquitecturas más clásicas dentro del campo de los sistemas inteligentes, que postulan la necesidad de técnicas de razonamiento a más largo plazo sobre un modelo del mundo [2]. Este tipo de arquitectura, con un módulo de alto nivel que toma las decisiones a largo plazo, obliga a que el robot tenga una representación interna del mundo donde realiza las acciones. El proceso de razonamiento a alto nivel trabaja sobre resultados intermedios de dicha representación, siendo ésta la única manera posible de solucionar problemas complejos.

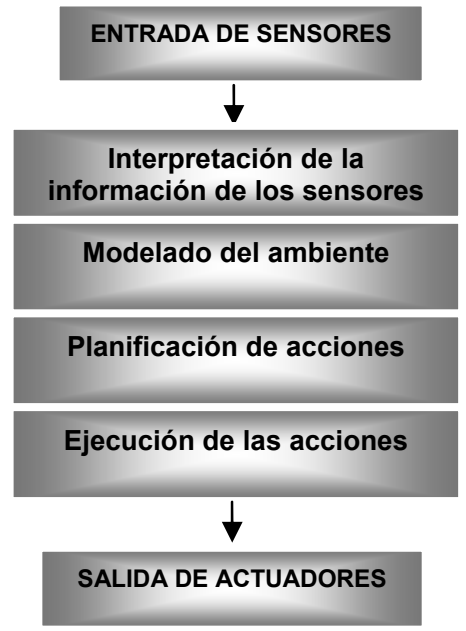

Fig. 2. Arquitectura basada en Conocimiento.

En cualquiera de los dos casos existe una tercera perspectiva que trabaja con el problema de la integración de múltiples robots para lograr la realización de metas complejas. El aspecto cooperativo, dado un sistema multiagente de 
robots, aparece en los dos tipos de arquitectura como un tercer nivel encargado de los aspectos de comunicación [5].

Siguiendo esta línea, el proyecto enfoca la integración de tecnologías de aprendizaje automático, redes neuronales, lógica difusa, algoritmos genéticos, computación evolutiva y sistemas inteligentes autónomos en el campo de la robótica cognitiva [1] [4]. Las metas del uso de dichas tecnologías varían desde el aprendizaje de modelos del entorno para realizar planificación reactiva, hasta la adquisición automática de heurísticas que controlen planificadores. Asimismo, se prevé la comparación de técnicas híbridas con técnicas evolutivas basadas en algoritmos genéticos y funciones de aptitud a efectos de comparar su efectividad en el diseño de la estructura cognitiva del robot.

\section{OBJETIVo DEL PROYECTO}

El principal objetivo de este proyecto es diseñar y ensayar diferentes arquitecturas de software donde coexistan módulos que permitan definir aspectos de comportamiento de robots autónomos y manipuladores, buscando maximizar aquellos parámetros que delimiten un desempeño deseado del robot en términos de sus prestaciones e interacción con el medio ambiente.

\section{Metodología Del Desarrollo}

A efectos de estudiar la relación entre la arquitectura neuronal del robot y su comportamiento en distintos ambientes y en el desempeño de diferentes objetivos de navegación, se procederá a utilizar el paquete de software MATLAB, versión 12.0, (con utilización del Toolbox de Neural Networks, Genetic Algorithms y Fuzzy Logic) tanto para la construcción del entorno como del agente robótico propiamente dicho, tal como se observa en la figura 3.

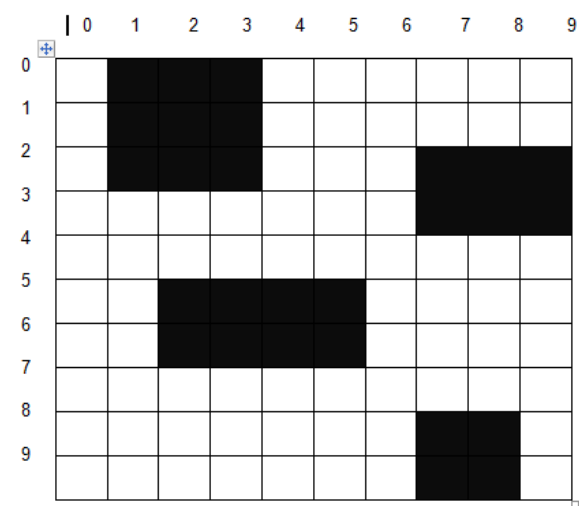

Fig. 3. Entorno de Navegación.

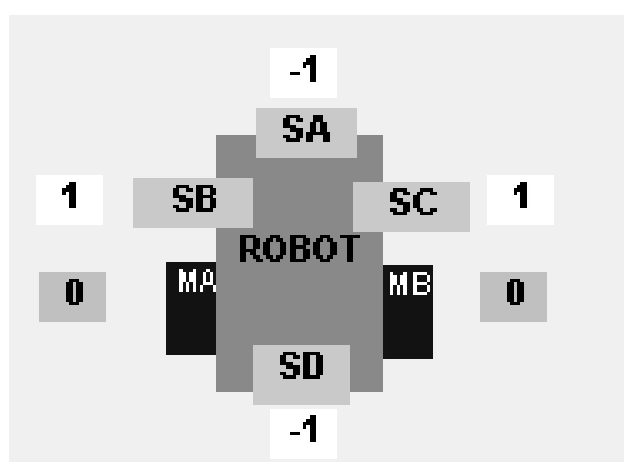

Fig. 4. Diseño del robot.

El esquema presentado en la figura 3, indica un entorno cuyos obstáculos se reducen a recuadros negros dentro de la grilla, cuyo ordenamiento corresponden a un eje cartesiano, a fin de posicionar al robot, donde los espacios en blanco representan lugares habilitados para el desplazamiento del robot. Más abajo, en la figura 4, puede observarse un robot tipo, dotado de cuatro sensores de obstáculos, esto es SA, SB, $\mathrm{SC}, \mathrm{SD}$, dos sensores de posición, PX y PY y dos motores o actuadores, los que le permiten desplazarse, MA y MB [3].

Los resultados de las simulaciones serán implementados en sistemas microcontrolados. Se prevén dos tipos de hardware. Uno será orientado a propósitos generales dedicado al manejo de motores y control básico. Este sistema incluirá manipulación de señales analógicas preveniente de los sensores e interfase de salida de potencia. Además se incluirá un transceptor en $2.4 \mathrm{Ghz}$ para el enlace con un sistema de alto nivel que permitirá realizar el data logging para las tareas de depuración y verificación de la simulación.

La segunda estructura de hardware, se construirá basada en DSC Digital Signal Controllers. Estos dispositivos combinan la potencia de cálculo de los DSP junto con las interfases analógicas y digitales necesarias en implementaciones robóticas. Los algoritmos de mayor exigencia de procesamiento en tiempo real serán implementados en este tipo de hardware. También incorporará un transceptor de 2.4 Ghz para las tareas de data logging mediante una computadora personal.

Se estudiará la mutua dependencia entre arquitectura de la red neuronal y complejidad de las trayectorias. Se tratará de encontrar pautas para el diseño de la red neuronal. Se le incorporarán al agente robótico módulos de memoria y de detección de condiciones de lazo cerrado. La evaluación de performance del agente se basará en la evitación de colisiones tanto para obstáculos fijos como móviles y en el cumplimiento de determinados objetivos básicos de comportamiento, como por ejemplo trasladar un objeto desde un punto a otro del entorno de navegación.

A continuación, se procederá a evaluar el comportamiento del robot cuando se lo programa empleando técnicas del aprendizaje automático, basadas fundamentalmente en al algoritmo ID3 de Quinlan [9], al que se lo adaptará para una versión en código MATLAB. Para evaluar el grado de efectividad del programa diseñado se lo comparará con los resultados arrojados por la herramienta de software Ctree. El aprendizaje automático permitirá extraer del árbol generado por el mismo las reglas de aprendizaje que describen el comportamiento del robot bajo este paradigma. De este modo, al ingresar un patrón de entrada, se obtendrá el valor de salida asignado al mismo conforme a este modelo. Es decir, dada una situación del robot en el ambiente, el paradigma basado en aprendizaje automático indicará la acción a seguir.

A efectos de comparar la performance de los paradigmas redes neuronales y aprendizaje automático tomados individualmente, se procederá a realizar distintos experimentos con posiciones de partida aleatorias. Se elaborará una función de aptitud que tenga en cuenta el número de colisiones y el número de situaciones de lazo cerrado con relación al número de experimentos realizados a efectos de analizar la efectividad de cada técnica cuando se la emplea por separado. Se busca conocer cuál de los dos métodos de programación permitió alcanzar la meta un mayor número de veces en el menor tiempo de simulación posible.

Con posterioridad, se procederá a hibridar la composición de la estructura cognitiva del robot conforme a un modelo jerárquico de capas, asignando conductas modeladas mediante redes neuronales (paradigma reactivo) a las capas de comportamiento más básicas y conductas de tipo más 
abstracto (paradigma del aprendizaje automático) a las capas superiores. Se estudiará que conductas es propicio asignar a cada capa, como así también sus características de diseño e interacción con las otras capas. Se realizarán experimentos con esta técnica de programación y se procederá a evaluar la efectividad de los diferentes paradigmas utilizados en términos de parámetros indicativos de: precisión, eficiencia, robustez y complejidad.

Una vez efectuada la comparación de las ventajas y desventajas de las distintas técnicas (redes neuronales, aprendizaje automático e híbrida) se procederá a agregar más capas a la estructura cognitiva del robot conteniendo estrategias de conducta superiores orientadas al cumplimiento de objetivos y sub-objetivos de comportamiento.

\section{Contribucion Al Avance Del Conocimiento CiENTíFICO Y/O TECNOLÓGICO}

La robótica es un área científica y tecnológica que en su definición más amplia abarca sistemas físicos, en general articulados, que son reactivos a condiciones no estructuradas. La robótica ha evolucionado de ser un área liderada por profesores de las facultades de mecánica en los cincuentas, pasando por la electrónica-eléctrica en los ochentas, a una disciplina con méritos propios, considerada independiente de las ciencias aplicadas.

La manufactura es una actividad tecnológica que realiza la transformación de materias primas, por medio de labor física o automatizada, en productos terminados para su venta, especialmente considerando procesos de producción a gran escala. La investigación en esta área es de naturaleza compleja que requiere para su buena ejecución de diversas disciplinas asociadas tanto a la tecnología de los productos y de los sistemas de producción, como a las áreas administrativas de distribución y comercialización.

La manufactura moderna tiene diferentes áreas de desarrollo que estudian y enfrentan problemas a diferentes niveles del proceso de producción, esto es, desde el nivel más bajo consistente en la elaboración de componentes simples, pasando por sistemas ensamblados compuestos, sistemas complejos de producción y distribución, hasta las estrategias operativas de todo un sector corporativo. En todos estos niveles se innova con el establecimiento de estrategias de diseño basadas en el modelado, simulación y control, para ayudar a la toma de decisiones. Particularmente, a partir de los noventas se ha dado una mayor importancia a la investigación de fenómenos encaminados a la mejora de los medios de producción por medio de la organización racional de tareas y de los medios de suministro, esto es, calidad total, manufactura esbelta, sistemas integrados de manufactura por computadora, sistemas flexibles de manufactura, etc.

El presente proyecto de investigación se fija como objetivo la obtención de estrategias de diseño ideales para las arquitecturas hardware-software de agentes robóticos tanto móviles como industriales, en función de las conductas y rendimiento deseados para el robot. Por lo tanto, su contribución a la generación de conocimiento científico y tecnológico está directamente relacionada con el desarrollo de proyectos para la construcción de robots desde un punto de vista innovativo. una extraordinaria competitividad y productividad, sino que también trasmiten una imagen de modernidad. En los países desarrollados, las inversiones en tecnologías robóticas han crecido de forma significativa y muy por encima de otros sectores. No obstante, el conocimiento sobre robótica de la mayoría de la sociedad sigue siendo muy limitado.

La robótica tiene como intención final complementar o sustituir las funciones de los humanos, alcanzando, en algunos casos, aplicaciones masivas. En el contexto industrial, donde los robots se utilizan con notable éxito desde hace varias décadas, los beneficios empresariales y sociales del presente proyecto se pueden resumir en cuatro aspectos:

- Productividad: aumento de la producción y reducción de los costos, sobre todo laborales, de materiales, energéticos y de mantenimiento.

- Flexibilidad: permite la fabricación de una familia de productos sin la necesidad de que se modifique la cadena de producción y por consiguiente, sin paradas ni pérdidas de tiempo.

- Calidad: debido al alto nivel de repetitividad de las tareas realizadas por los robots, las mismas aseguran una calidad uniforme del producto final.

- Seguridad: dado que los procesos de fabricación se llevan a cabo con un mínimo número de personas, se disminuye la posibilidad de accidentes laborales y se reemplaza a operarios en la ejecución de tareas tediosas o peligrosas.

\section{CONDICIONES InSTITUCIONALES PARA El DESARRollo Del Proyecto De InVESTIGACIÓN}

Este proyecto articula líneas de investigación en el área de Robótica del Laboratorio de Electrónica de la Facultad Regional del Neuquén en articulación con el Departamento de Ciencias Básicas de la misma casa de altos estudios, a cargo del Director propuesto. Las líneas de investigación del área cuentan con financiamiento de la Secretaría de Ciencia y Técnica de la misma Universidad.

\section{REFERENCIAS}

[1] García Martínez, R. Sistemas Autónomos. Aprendizaje Automático. 170 páginas. Editorial Nueva Librería. ISBN 9509088-84-6. 1997.

[2] García Martínez, R., Servente, M. y Pasquini, D. 2003. Sistemas Inteligentes. 347 páginas. Editorial Nueva Librería. ISBN 9871104-05-7.

[3] Adrian. E. Scillato, Daniel. L. Colón y Juan. E. Balbuena. Técnicas de Navegación Híbrida para Navegación de Robots Móviles. Ed. Rama de Estudiantes del IEEE. Tesis de grado para obtener el grado de Ingeniero Electrónico. Universidad Nacional del Comahue. (2005)

[4] Sierra, E. Hossian, A. Britos, P. García Martínez, R. Fuzzy control for improving energy management in indoor building environments Proceedings del Congreso de Robótica Electrónica y Mecánica Automotriz, CERMA 2007. IEEE. Morelos (Cuernavaca), México.

[5] Sierra, E. Hossian, A. Britos, P. García Martínez, R. A multiagent intelligent tutoring systems for learning computer programming. Proceedings del Congreso de Robótica Electrónica y Mecánica Automotriz, CERMA 2007. IEEE. Morelos (Cuernavaca), México.

\section{CONTRIBUCION AL DESARROLLO SOCIO-ECONÓMICO}

Para una sociedad, la robótica es sinónimo de progreso y desarrollo tecnológico. Los países y las empresas que cuentan con una fuerte presencia de robots no solamente consiguen 


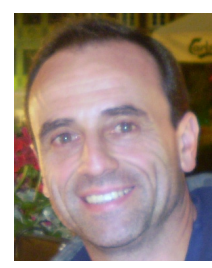

Alejandro Armando Hossian. Es responsable de formar recursos humanos en el área de investigación y desarrollo dirigiendo tesis de doctorado y maestría vinculadas a sus áreas de especialización. Es Profesor Titular Regular de la Facultad Regional Neuquén, Universidad Tecnológica Nacional (UTN - FRN). Es Docente Investigador, Director del grupo de investigación de robótica cognitiva y Director de Posgrado de la misma casa de altos estudios. Es Docente Investigador Categoría III del Ministerio de Educación y Par Evaluador de la Comisión Nacional de Evaluación y Acreditación Universitaria (CONEAU), Republica Argentina. Acredita estudios universitarios de Ingeniero Civil por la Universidad Católica Argentina, es Especialista en Ingeniería de Sistemas de Expertos por el Instituto Técnico Buenos Aires (ITBA), es Máster en Ingeniería de Software por la Universidad Politécnica de Madrid (UPM), es Magister en Ingeniería de Software por el Instituto Técnico Buenos Aires (ITBA) y es Doctor en Ciencias Informáticas por la Facultad de Informática de la Universidad Nacional de La Plata (UNLP).

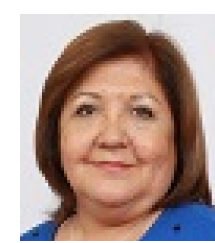

Lilian. Cejas. Posee titulo de grado universitario de Ingeniera Química, una Especialización en Docencia Universitaria y Maestría en Docencia Universitaria. Se ha capacitado en distintos cursos afines a su desempeño profesional enfocado principalmente al área educacional superior universitario. Se ha desempeñado desde hace más de veinticinco años en la Universidad Tecnológica Nacional, Argentina. Es docente-investigadora en la modalidad presencial y desde unos diez años en la modalidad virtual, como docente, investigadora. Es Directora de Departamento de Ciencias Básica y Directora de Carrera de la Licenciatura en Tecnología Educativa, modalidad a distancia. Participa como docente invitada en la modalidad virtual de la Universidad Autónoma del estado de Hidalgo, México. Ha participado en numerosos congresos y reuniones científicas nacionales e internacionales, tanto con presentación de trabajos de investigación como organizadora, como así también es miembro de comisiones científicas y tecnológicas.

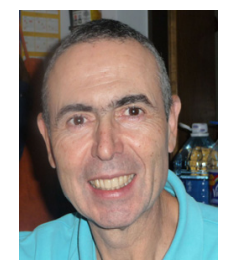

Roberto Carabajal. Egresado como Ingeniero Electricista orientación Automática, se ha especializado en software para la administración y control en la industria, con amplia experiencia en redes, servidores y bases de datos. Ha trabajado como proveedor de servicios en el área del mantenimiento electrónico para informática. Fue profesor en la carrera corta Técnico Superior en Programación de la Facultad Regional del Neuquén, y actualmente se desempeña como profesor en ingeniería química y electrónica de la misma facultad. Está categorizado con Categoría "5": Programa de Incentivos a docentes e investigadores de Universidades Nacionales y con Categoría "E": Carrera de docente investigador de UTN. Ha participado en diversas publicaciones científicas y en el grupo de investigación Desarrollo de Métodos y modelos no convencionales para la programación de comportamientos en robots. Es codirector de la obra de diseño y puesta en marcha de la planta Planta PilotoUnidad de Destilación de Dos Componentes de la F.R.N.

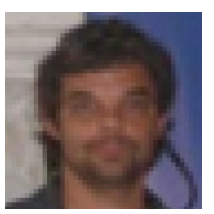

Carlos César Echeverría. Ingeniero Electrónico por la Universidad Nacional de La Plata. Docente de la Universidad Tecnológica Nacional Facultad Regional de Neuquén en las cátedras de Teoría de Circuitos 2, Electrónica Aplicada 1 y Medidas Electrónicas 1. Categorizado con Categoría " 5 " del Programa de Incentivos a docentes e investigadores de Universidades Nacionales y con Categoría "E" de la Carrera de docente investigador de UTN. Profesor en Disciplinas Industriales (PDI) - Profesor en Docencia Superior (PDS). Diplomatura Superior en Enseñanza de las Ciencias. Especialización en "Tecnología de antenas para uso Satelital". Ha participado en diversas publicaciones científicas y en el grupo de investigación Desarrollo de Métodos y modelos no convencionales para la programación de comportamientos en robots.
Ha desarrollado varios proyectos sobre la implementación de tecnología en las aulas de enseñanza de Ingeniería y ciencias duras como la Física y la Matemática.

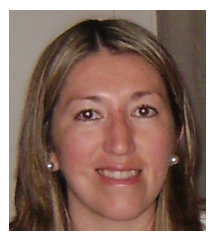

Lorena Verónica Olivera. Trabaja en el grupo de Investigación de Robótica de la Facultad Regional del Neuquén de la Universidad Tecnológica Nacional (UTN-FRN). Docente Investigador por la Universidad Tecnológica Nacional. $\mathrm{Ha}$ participado en la elaboración de artículos sobre automatización y Robótica Cognitiva. Es Ayudante de la Cátedra de "Tecnología de redes neuronales" en UTN - FRN. Es Estudiante avanzado de Ingeniería Electrónica en la misma casa de altos Estudios.

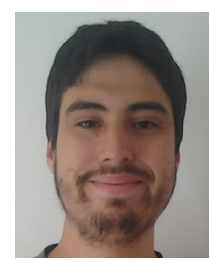

Emanuel Maximiliano Alveal. Becario de investigación que desarrolla sus actividades en el marco del Grupo de Investigación en Robótica de Manipuladores de la Universidad Tecnológica Nacional - Facultad Regional del Neuquén. Estudiante avanzado de la Carrera de Ingeniería Electrónica de la misma casa de altos estudios. 